\title{
Regimes of self-organized criticality in the atmospheric convection
}

\author{
F. Spineanu, M. Vlad, D. Palade \\ National Institute of Laser, Plasma and Radiation Physics \\ Bucharest 077125, Romania
}

June 19, 2018

\begin{abstract}
Large scale organization in ensembles of events of atmospheric convection can be generated by the combined effect of forcing and of the interaction between the rising plumes and the environment. Here the "large scale" refers to the space extension that is larger or comparable with the basic resolved cell of a numerical weather prediction system. Under the action of external forcing like heating individual events of convection respond to the slow accumulation of vapor by a threshold-type dynamics. This is due to the a time-scale separation, between the slow drive and the fast convective response, expressed as the "quasi-equilibrium". When there is interaction between the convection plumes, the effect is a correlated response. We show that the correlated response have many of the characteristics of the selforganized criticality (SOC). It is suggested that from the SOC perspective, a description of the specific dynamics induced by "quasiequilibrium" can be provided by models of "punctuated equilibrium" - Indeed the Bak-Sneppen model is able to reproduce (within reasonable approximation) two of the statistical results that have been obtained in observations on the organized convection.

We also give detailed derivation of the equations connecting the probabilities of the states in the update sequence of the Bak-Sneppen model with $K=2$ random neighbors. This analytical framework allows the derivation of scaling laws for the size of avalanches, a result that guves support to the SOC interpretation of the observational data.
\end{abstract}




\section{Introduction}

Many natural systems consist of large collections of identical sub-systems that do not interact one with another. In many cases the ensemble (i.e. the whole system) is subject to an external drive at a rate which is much slower than the corrective reaction of any sub-system when it becomes unstable. In some circumstances the system evolves to a type of behavior called "Self Organized Criticality" (SOC). To introduce the basic terminology we start with a simple example: an inflammable gas emerging with a slow rate at the flat surface of a porous material. The burning occurs when the fraction of density of inflammable gas in the air reaches a threshold, at the surface of the porous body. Due to the porosity the gas will accumulate at random at the surface, eventually creating patches of gas that emerged from neighbor pores. If above one of such pore there is ignition, it propagates very rapidly (like an avalanche) to all the sites within the patch, burning the gas and resetting to initial state. Patches that are spatially separated (i.e. non-connex) are not affected. The dimensions of the patches (equivalently, of the avalanches of burn) are arbitrary and can be as large as the whole surface of the porous material. This state is similar to the criticality, without however the system to undergo any phase transition. The state is stationary in the statistical sense. The fluctuations (local burning events) are correlated in space and in time with dependences on space and respectively time intervals exhibiting long range algebraic decays. As simple as it is, this system introduces basic elements: slow rate of feeding, threshold, fast reaction in each column, avalanches. These are elements that describe one of the most fundamental way of organization of systems in nature.

Each sub-system has a behavior that is characterised by a threshold. The threshold refers to a parameter (like: density of inflammable gas, height of a column in a sand pile, amount of water vapor in a convective column, etc.) and separates the regime of quiet equilibrium from the active regime. The activity consists of a fast instability (burning, toppling of sand grains from the column, precipitation from the convection, etc.) that returns the subsystem to the equilibrium regime, in general by removing a certain amount of substance, of the same nature as the drive: gas, sand, water, etc. This is transmitted to neighbors and those that are close to threshold can themselves switch to active state, etc. This chain of influences triggered by the instability of one initial sub-system is fast like an avalanche and involves a set of subsystems that were all close to threshold. It only stops when the neighbor sub-systems are far from threshold and even if they receive the pulse from the active ones they cannot reach the active regime. The "substance" is redistributed among the sub-systems. This picture can easily be generalized 
to situations where there is no "substance" that is transfered between the sub-systems. For example, a sub-system that switches to active state can undergo an internal re-organization that reinstates back the equilibrium and it simply emits a signal. Neighboring sub-systems that receive the signal and are themselves close to threshold will switch to active state and undergo internal re-organization, emitting signals, etc.

The drive originates in an external source (e.g. the radiative heating of the land) and may affect one or several sub-systems, chosen at random. Equally possible, the source can drive the whole ensemble of sub-systems, uniformly or not, but always at a rate which is slow relative to the fast reaction of any sub-system when the threshold is exceeded. Under the weak drive the system evolves slowly to a state in which most of the sub-systems are close to threshold. The further slow "drive" will produce avalanches of various sizes that return the sub-systems to the equilibrium state (i.e. under the threshold). The system as a whole preserves this state as statistical stationarity. This state is very similar to the state of a system that is on the point to make a phase transition. The size of an avalanche is similar to the length of correlation of the fluctuations. The avalanches can be extended over all spatial scales, up to the dimension of the system and in this analogy a correlation length which involves all the spatial extension of the system is the signature of criticality. In the case of SOC the system does not make a phase-transition but stops at the critical state. The SOC state is statistically stationary so it is energetically ideal : the activity consists of random transients (avalanches) and the system explores the space of states specific for criticality. The system now acts under a rule: minimum rate of entropy production. It just reacts to external "excitation" such as to keep this statistical equilibrium.

\section{Classical view on SOC}

During the slow feeding many sub-system can reach the marginal stability, without however becoming active. The existence of a threshold means that the state of activity is separated from the last "bound" state, the marginally -stable one, by an interval. A simple fluctuation, with an amplitude comparable with this gap, will switch the sub-system from marginal stability to active state. There are many systems that exhibit SOC. Models are of the types: (a) algorithmic, like sand pile, Bak - Sneppen, described by a set of rules for advancing in time the components (sub-systems); (b) analytic: like the Kardar Parisi Zhang equation describing gradient drive $\mathbf{v}=-\nabla h$ for a 
scalar field $h$ that may represent the local accumulation of a quantity (dust falling randomly on the bottom of a river). For this case the approach is based on the Dynamic Renormalization Group. Very good references exists on the SOC subject (Bak, 1996; Sornette, 2006; Jensen, 1998).

\section{The SOC of the atmospheric convection pro- cesses}

The physical processes that take place inside the grid cell of a finite resolution numerical model have a fundamental role in the success of the large scale dynamics simulation. This is the problem of parameterization, still under active research. Part of the difficulty comes from the fact that there is a wide variety of situations at the small scales which would have to be represented. The diversity of physical states cannot be simply reduced to few global charactersitics whose formal description would allow to transfer reliable quantities to the large scale dynamics. Different physical situations require different formalisms, with various weights placed on the component of the small scale description: convection (either shallow or deep), cloud distribution, entrainment mechanisms (which are dominated by turbulence with different characteristics), detrainment, downdraft and effects on low level convergence, etc. We should probably admit that, instead of a single formalism for parameterization, one must consider a variety of formalisms, each adapted to the characteristics that are dominant at a certain state of the atmosphere in the small scale region. However this is a heavy theoretical task. It first requires to reduce the diversity of physical situations to a finite number, ennumerated and characterized in a systematic way. Second, one should find a way to identify which particular "behavior" is manifested such as to activate the adequate formalism, prepared for that particular "behavior". This is a difficult programme, but one can recognize that the stochastic parameterization seems to partially fulfill this task, since by definition it explores a variety of states which belong to the statistical ensemble of realizations of a field of random physical variables. An interesting dynamics is revealed by the numerical studies based on cellular automata (Bengtsson et al., 2013).

This introduction is intended to suggest that in connection with the search for a reliable parameterization (and implicitely for the successful large scale numerical modeling) one needs to explore the diversity of physical situations which occur in limited areas and draw conclusions on how different the formalism can be for the description of each of them.

It is not sure that this approach can be realized in practice. However it 
produces interesting and possibly useful secondary results. This is because it suggests to abandon the search for a unique formalism and encourrage to first investigate the typologies of "behaviors" that can be identified as sufficiently individualized and distinct. Applying this idea and looking for general characteristics of evolutions (connecting small scales, of the order of grid cell, and the large scales) one can identify in some circumstances systematic elements which point to the large scale organization of convective events. Many observational studies support this conclusion, but a unifying concept that should be found behind this manifestation has not yet been formulated.

Obviously, the large scale organization of convective events is not the unique possible state of the atmosphere. Strong drive inducing large scale response consisting of strong horizontal pressure gradients, generation of vortical flows, jets and in general displacement of masses of air over large distances do not fit into the characteristic state of SOC (at least in its most common, simple, picture) and need different approaches. The organization of the convection in the way that can be described by SOC apperas to be just one of the possible states of the atmosphere and as such SOC cannot claim to become the unique, paradigmatic reference of atmosphere dynamics. But it is relevant in some particular situations and this is supported by the fact that correlations of fluctuations of physical quantities show algebraic decay both in space and in time. The basic elements mentioned before as specific to SOC are a natural component of the large scale convection systems. The "quasi-equilibrium" hypothesis recognizes the presence of two largely separated time scales: the slow external forcing and the fast convective response, which is similar to the corresponding property of the sub-systems' dynamics in SOC. The mutual influence between the sites of convection is also similar to the avalanches.

The important aspect of large scale organization of the convection and precipitation has been revealed, in a more or less explicit form, in many works. In particular, it has been accumulated evidence of a mesoscale organization of systems of clouds. Leray and Houze (1979) study the genesis and evolution of a tropical cloud cluster. In the formation phase the spatial characteristic consists of a line of isolated cumulonimbus cells whose orientation is transversal on the direction of the low level wind. Further, rain areas within individual cells merge. In this period new convective cells are generated between and ahead of the existing cells. This is explained by the downdrafts originating from old convection cells, which enhance the convergence at low levels. This provides moist flux to new cumulonimbus updrafts, enhancing their buoyancy, and producing new convection cells. While the convection 
cells will eventually dissipate, new other convections are generated. This is a propagation, in which the new convective cells develop in front of the line of advancing precipitation system, faster than the dissipation of the older cells at the rear. We underline the effect that a localized convection exerts on the neighbor sites, this being compatible with the idea that there can be propagation of an effect in an ensemble of sites, as in an avalanche specific to SOC. The role of mutual trigger is played by the downdraft from previous active convection sites. The cloud cluster is characterized, at later times, by the persistence of a large area of precipitation behind the advancing front.

From this complex picture we focus on the aspects related to the propagation of influences between sites of convection, which bear some analogy with the avalanche phenomena and seem to support the concept of selforganization at criticality. We also note a particularity, that propagation in systems of convections located at nearby sites needs a finite time interval, while in many classical realizations of SOC the avalanche is taken as simultaneous in all sites that are involved.

The formation of "clumps" of clouds has been discussed by Lopez (1977), pointing out the random growth before merging into large scale mesoscopic formations. We note then that the SOC concept of avalanche, which is a correlated behavior of a set of sub-systems that are all at, or close, to the marginal stability and switch to active state by the effect of a influence coming from a nearby site, must here be adapted and become the signature of the process of generation of large scale formation of clouds out of isolated active sites of convection. The resource of active convection are stimulated by mutual influence.

The paper of $\mathrm{Su}$, Bretherton, and Chen (2000) on self aggregation is a study of the generation of large clusters from isolated convections. Various scenarios have been proposed, e.g the "gregarious" convection (Mapes, 1993) or, the wind-induced surface heat exchange (Emanuel, 1987), (Neelin et al., 1987). The large scale organization of convection events has a multiple manifestation but at least the first phase seems to strongly suggest SOC. The initially distinct convections interact through the subsiding air between them and within an interval of approximative ten days they organize into mesoscale patches of rainy air columns. Further, the mesoscale patches of each type (rainy and respectively dry) coalesce generating a single moist patch surrounded by dry subsiding air. Except for the final spatial distribution the large scale propagation of mutual influence is similar to the generation of an avalanche in a SOC system.

A description of this propagation is offered by Cruz (1973): "The radar observed progression of one hot tower is a sequence of growing deep cumuli one ahead of the other in the direction of the cloud motion". 
In more precise terms, the influence consists of the change of the environment properties in the region of the nearby site. This is done by downdraft and precipitation.

There is a minimum time for the process of interaction to take place: the development of the convection at the initial site, approximately half an hour, the decay of this convection in another half of hour, the thermal influence of the conditions for the nearby site through modification of the vapor content and of the temperature of the air. We can take as an elementary measure of time the interval $\delta t \sim 1$ hour. For comparison Plant and Craig (2008) have adopted a duration of an individual plume $\delta t=45 \mathrm{~min}$. It is defined the closure time scale $T_{c}$, called the adjustment time in response to a forcing. In this interval $90 \%$ of the convective available potential energy (CAPE) would be removed if the ensemble of plumes were acting on the environment.

Now we can be interested in an estimation of the average distance between nearby sites where convection may arise, if local conditions are favorable. The distance between clouds, for densly packed states can be taken as the average dimension of the cloud, assumed to be $\delta l=2 \mathrm{~km}$. We can use this input to estimate a speed for the propagation of the non-material influence which consists of the fact that the convection at one site may trigger convection at the nearby site

$$
v_{\text {prop }} \sim \frac{\delta l}{\delta t}=2 \mathrm{~km} / \text { hour }
$$

This speed is certainly different of the velocity of propagation of gravityinertial waves between clouds. What is important is the fact that the SOC picture defines a new type of propagation, which is different of the usual gravity-inertial waves generated through geostrophic adjustment. However this propagation does not have a unique direction and does not transport momentum or energy. Is just a trigger for convection in nearby sites.

Several important works have underlined the relevance of SOC for the atmospheric convection and have provided solid arguments. Specific to this field, they consist of identifying algebraic decay of correlations. Peters et al. (2001) examines the statistics of the size of a precipitation event, $M$. The number of events of size $M$ is

$$
N(M) \sim M^{-1.36}
$$

Another observation (Peters et al., 2002) regards the duration between precipitation events (inter-occurrence time), which is given in function of the duration $D$ in minutes

$$
N(D) \sim D^{-1.42}
$$


We note again a close similarity with the SOC model (Bak - Sneppen) where the exponent is $3 / 2$.

Peters and Neelin (2006) propose for the intense precipitation events a picture inspired from phase transitions, with the water vapor $w$ as the tunning parameter (like the temperature in the magnetization) and the precipitation rate $P(w)$ as the order parameter. The slow drive is the surface heating and evaporation. The fast dissipation of buoyancy and rain water, is the precipitating convection. This "fast dissipation by moist convection prevents the troposphere from deviating strongly from marginal stability". This maintains the quasi-equilibrium which is the basic postulate of the Arakawa Schubert parameterization (Arakawa and Schubert, 1974).

After the critical threshold, the statistical averages have variations with the tuning parameter $w$ as

$$
\langle P\rangle(w)=a\left(w-w_{c}\right)^{\beta}
$$

where $\beta$ is an universal exponent. After scaling with factors that are imposed by the different climateric regions considered, the variation of the averaged probability $\langle P\rangle$ with the difference $\left(w-w_{c}\right)$ shows the same slope

$$
\log \langle P\rangle \sim \beta \log \left(w-w_{c}\right)
$$

with a slope $\beta \sim 0.215$.

Peters, Neelin, and Nesbitt (2009) analyse the mesoscale organization of convective events from the point of view of similarity with the statistics of percolation. The essential element that underlies the large scale organization is a local property: the sharp increase in the rate of precipitation beyond a certain value of the water-vapor content of the air column. This sharp threshold and the fast reaction that follows (precipitation) are seen as elements composing the usual scenario of a first order phase transition, in a continuous version. Since the distinctive component of the large scale organization in a limiting regime is the generation of a cluster (of convective events) of the size comparable to the system's spatial extension, it has been assumed that this is analogous to the percolation in a two-dimensional lattice of random bonds.

Although this is a very solid argumentation in favor of a kind of phase transition we have reserve to the proposed classification of this state as a phase transition. Indeed there are arguments to place the large scale organization of convective events in the same universality class as the percolation in $2 D$. However we should remember that the SOC itself, as represented, for 
example, by the sand pile, belongs to the universality class of the percolation. This is interesting in itself but does not cover all possible interpretations that can be associated to the power law dependence of the correlations of the fluctuating fields in a cluster of convections. We find more appropriate to associate the generation of a correlated cluster of convection that covers almost all the space domain investigated, - with the divergence of the susceptibility, but, recognizing simultaneously that correlations on all spatial scale are also possible. Equivalently, we find an approximative state of criticality for the system. Since however a phase transition, to a completely new phase, is not - and cannot be seen, we identify this state as the statistical stationarity specific to the self-organization at criticality.

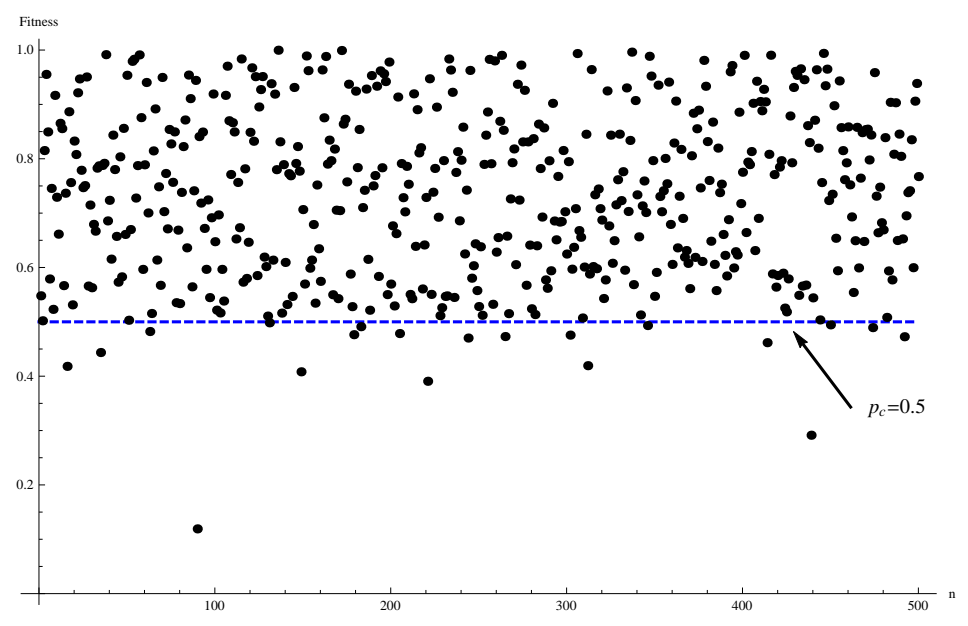

Figure 1: Simulated avalanche in the Bak-Sneppen model in 1D with one random site updated; the simulation uses $n=500$ species; first true avalanche appears after $\sim 10^{3}$ updates from the initial homogeneous state.

Many factors, like the content of water in a column of air, the type of convection (shallow or deep), the spatial correlation through the effect of downdrafts on the environment, etc. contribute to the regime of precipitation. Besides the complexity of the fluid and thermic processes it is still interesting to look for a low order dynamics that would be able to capture the essential aspects. A first suggestion comes from the fact that the statistics of cloud clusters exhibits multiple scale organization, whose protopype is the Continuous Time Random Walk. From the universality of classes of statistical ensembles of random processes, the rate of occurence of earthquakes has a similar property. The similarity between the statistical properties of cloud 


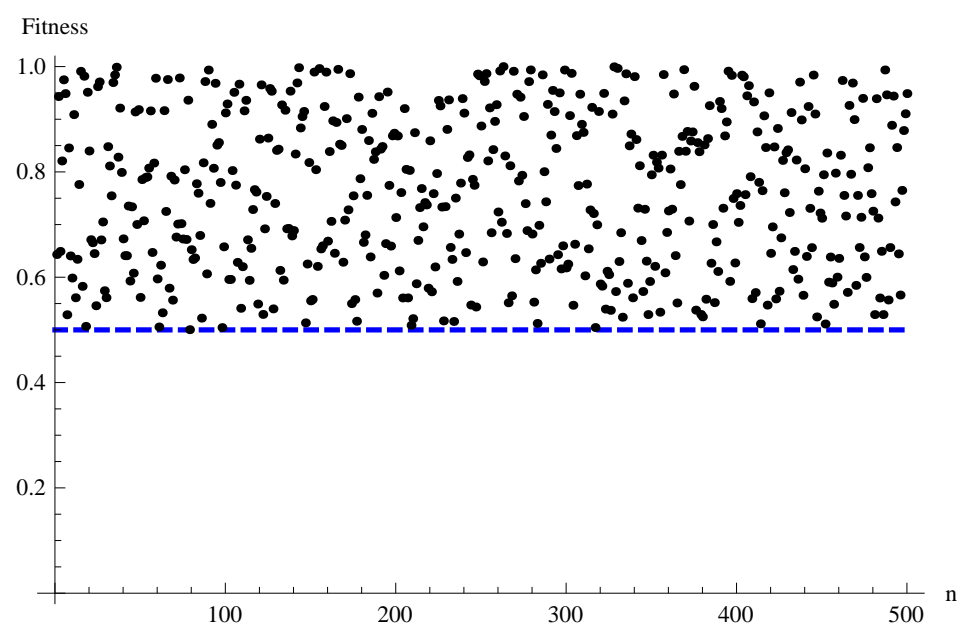

Figure 2: Numerical simulation of the critical state in the Bak-Sneppen model in 1D with one random updated site; the simulation uses $n=500$ species and in the large time limit the threshold $p_{c}=0.5$ it is reached (blue, dashed line)

sizes and of earthquake intensities has been noted and studied previously. It is then simpler to examine the way this statistics results from general properties of the tectonic breaking, with phases of accumulation followed by sudden release of energy. The known property of the earthquakes to exhibit self-organization at criticality is therefore indirectly made plausible, i.e. it extends to the similar ensemble of random precipitation events. Or, there is a standard reference for the dynamical behavior of the earthquakes in the state of SOC, the Bak - Sneppen model.

\section{The SOC formulation of convection within the Bak - Sneppen model}

\subsection{Why SOC realized in a "punctuated equilibrium" system may be relevant to the organization of the convection-precipitation events}

The model consists of an ensemble of sites (sub-systems) each characterized by a value of a parameter which reflects the degree of adaptation ("fitness") to the environment. There is a threshold level of fitness $\lambda$. By definition the sites whose current level of fitness is smaller than $\lambda$ are not in equilibrium 
relative to the environment and can possibly switch to an active state, which involves the update of their fitness parameter with a new, random, value, extracted with uniform probability from $(0,1)$. It is only certain that the site $k$ with the smallest fitness parameter $x_{k}$ (of course, $x_{k}<\lambda$ ) is subject to update and the other sites (both $<\lambda$ and $>\lambda$ ) are updated only if they are currently in a relationship of mutual influence with the site $k$.

The approach to the state of SOC in the atmosphere may be similar to the evolution of the gap in the Bak Sneppen model (Paczuski et al., 1996). The gap is the distance between zero level of fitness and the current average level of fitness, which is below the threshold $\lambda$. The gap tends to increase and the difference between the value of the fitness at a certain time and $\lambda$ decreases. The dynamical evolution of the gap consists of plateaux interrupted by fast events when there are avalanches. In time the gap approaches $\lambda$ which however it will only reach asymptotically.

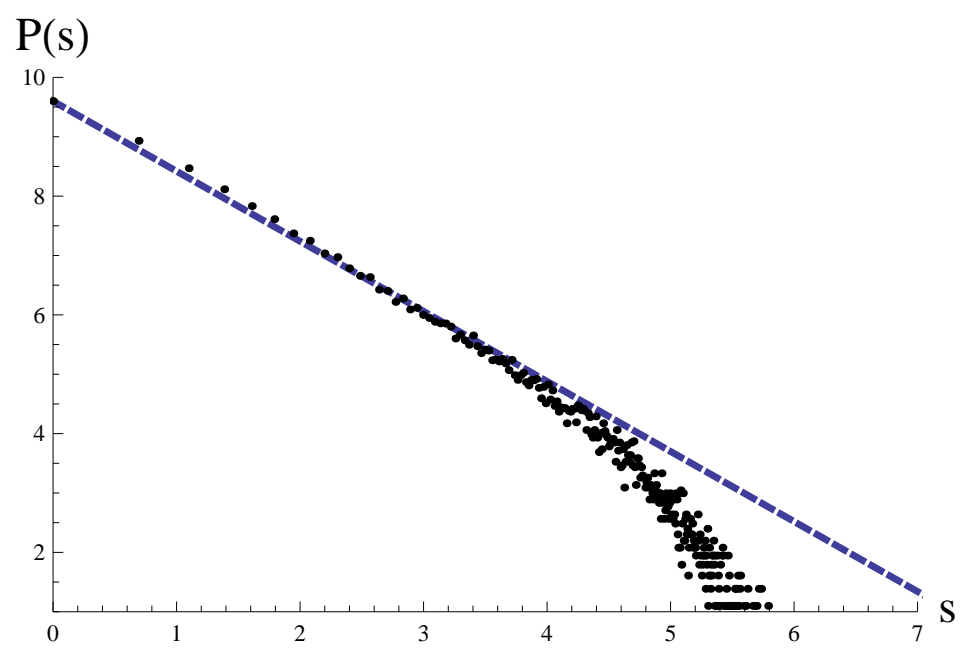

Figure 3: Numerical obtained correlation for avalanche statistics $\log (P(s))$ vs $\log (s)$ in the $1 \mathrm{D}$ Bak-Sneppen with one random updated site. The obtained exponent is $\simeq 1.32$ less than the analytical one due to finite $n$ and finite time of simulation.

The time evolution which precedes the SOC state therefore consists of the increase of the average fitness parameter across the system, or, in other words, the increase of the gap which tends to reach the $\lambda$ value. In atmosphere this regime corresponds to the time interval when there are long periods of inactivity interrupted by pricipitation events. The precipitation events remove part of the water vapor from a number of sites. This sub-ensemble of sites 


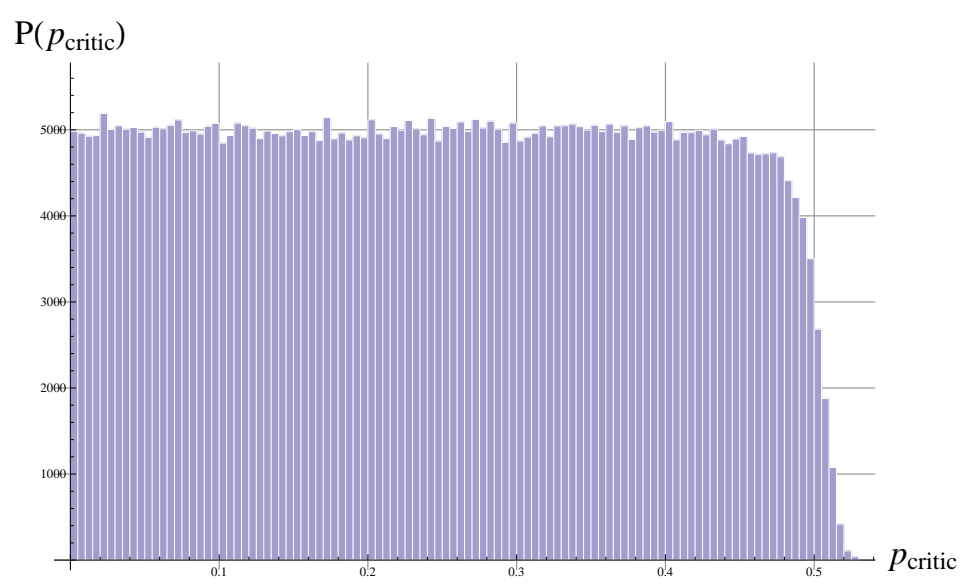

Figure 4: Histogram of the minim value of fitness of every time step in the 1D Bak Sneppen with random neighbor. The Heaviside-Theta like plot has the critical value at 0.5 as theoretical predicted. Simulation done with $n=50$ and $m=10^{6}$ updates

which are active almost simultaneously represent an avalanche. Affecting successively various sub-ensembles of sites the process removes part of the water vapor from almost all sites and the final result is that the differences in the amount of water vapor decreases progressively. The fact that the sites have similar values of this parameter (amount of water vapor) makes possible large scale responses of the system to a fluctuation of the drive, or, in other words, the avalanches can reach the spatial dimension of the system. This is the signature of the criticality. Reaching this state the system remains in a statistically stationary state.

This is a model of punctuated equilibium. By punctuated equilibrium one understands the situation where "any given small segment of the sites will experience long periods of inactivity punctated by brief periods of violent activity" (de Boer et al., 1995). The model is shown to describe the statistics of earthquakes (Ito, 1995).

\subsection{Probability of duration of correlated convection events}

We will use the statistics of avalanches for the Bak - Sneppen model. At the moment of update, one must identify the smallest barrier $b_{i} \in(0,1)$ and updates the site $i$. After updating the site $i$ one updates other $K-1$ sites, chosen at random. The model is called "random neighbors" (Paczuski et al., 
1996; de Boer et al., 1995, 1994).

Our comparison is based on the following mapping. The sites are local atmospheric processes (attached to a small and fixed area) that are close to produce convection. "Fitness" is a characteristic that refers to non-activity. A site fits to the conditions of non-activity when the conditions of convection are not realized. The sites (local atmospheric processes) are affected by the other sites, during their update. We now derive quantitatively few results regarding Bak-Sneppen statistics, that seem to be close to the statistics of the atmospheric processes.

Assume that system consists of $N$ sites, each characterized by a real number $x_{i}, i=1, N$. The dynamical rule is: at each time step the $x_{i}$ with minimal value is replaced by a random number extracted from the interval $[0,1]$ with uniform probability distribution. Together with $x_{i}$, other $K-1$ species are replaced by random numbers, in the same condition as $x_{i}$.

\subsection{The $K=2$ model of random neighbor: the algo- rithm}

At every time step (update) it is chosen the site with the minimum barrier $x_{i}$ and in addition $K-1$ other sites and the fittness parameters are updated. In this model only one other site, chosen at random, is updated (de Boer et al., 1994). The definition of an avalanche is made in the following steps: (1) fix a threshold barrier $\lambda$; (2) count the number of active sites, which means count the number of sites that have a barrier less than $\lambda$; (3) an avalanche of temporal duration $T$ is is said to occur when there are active sites for consecutive $T$ temporal steps. The duration of the avalanches has a probability with the scaling at large $T$

$$
P_{\text {aval }}(T) \sim T^{-3 / 2}
$$

There is a scaling low governing the time separation of the moments when the same site will be again the minimum from all the sites. The first return probability $S(t)$ is defined as the probability that, if a given site $i$ is the minimum at time $t_{0}$ it will be again minimum - but for the first time - at time $t_{0}+t$. This means that between $t_{0}$ and $t_{0}+t$ the site $i$ has not been minimum. The probability $S(t)$ for the random neighbor model, scales as

$$
S(t) \sim t^{-3 / 2}
$$

Important results on the Bak-Sneppen model can be derived analytically. We present here and in the Appendix results that have been obtained by Boettcher and Paczuski (1996) and de Boer et al. (1994). They permit to 
make a comparison with the results of Peters and Neelin (2006) in the atmospheric convection.

We fix a real value for a parameter $\lambda$. Consider the number $n$ of sites that have the value $x_{i}$ less than $\lambda$. Define $P_{n}(t)$ as the probability that at time $t$ there are $n$ sites that have value $x_{i}$ lower than $\lambda$. This probability verifies the following master equation

$$
P_{n}(t+1)=\sum_{m=0}^{N} M_{n, m} P_{m}(t)
$$

where (de Boer et al., 1995)

$$
\begin{aligned}
M_{n+1, n}= & \lambda^{2}-\lambda^{2} \frac{n-1}{N-1} \\
M_{n, n}= & 2 \lambda(1-\lambda)+\left(3 \lambda^{2}-2 \lambda\right) \frac{n-1}{N-1} \\
M_{n-1, n}= & (1-\lambda)^{2}+\left(-3 \lambda^{2}+4 \lambda-1\right) \frac{n-1}{N-1} \\
M_{n-2, n}= & (1-\lambda)^{2} \frac{n-1}{N-1} \\
M_{0,0}= & (1-\lambda)^{2} \\
M_{1,0}= & 2 \lambda(1-\lambda) \\
M_{2,0}= & \lambda^{2}
\end{aligned}
$$

Consider $N$ sites and fix the parameter $\lambda=1 / 2$. Consider that at time $t$ there are $m$ sites for which $x_{j}<\lambda$. The probability that at time $t$ there are $m$ such sites is $P_{m}(t)$. In terms of random walk, the start of an avalanche is the start of a walker at $x=0$. Later the walker stops as it reaches again 0 . This is the end of an avalanche. One defines $P_{2 n} \equiv$ probability that a walk will return to $x=0$ at step $2 n$. To find it one starts with a more general quantity: $Q_{2 n}=$ probability that a walk started at $x=0$ returns at $x=0$ irrespective of the fact that it returns to $x=0$ several intermediate times. This quantity is known in the theory of random walks

$$
Q_{2 n}=\frac{1}{2^{2 n}} C_{2 n}^{n}
$$

Since $Q_{2 n}$ is a collection of $P_{2 n}$, one can use the generating functions

$$
1+\sum_{n=1}^{\infty} Q_{2 n} z^{2 n}=\frac{1}{1-\sum_{n=1}^{\infty} P_{2 n} z^{2 n}}
$$


The left hand side is explicit and gives

$$
1-\sum_{n=1}^{\infty} P_{2 n} z^{2 n}=\sqrt{1-z^{2}}
$$

from where

$$
\begin{aligned}
P_{2 n} & =\frac{(2 n-3) ! !}{(2 n) ! !} \approx \sqrt{\frac{2}{\pi}} \frac{1}{(2 n)^{3 / 2}}(\text { large } n) \\
& \sim \tau^{-3 / 2}
\end{aligned}
$$

This is the scaling of the duration of an avalanche. This is not far from the result of Ole Peters Eq.(2) in the statistics of the atmospheric precipitation.

\section{The Gierer - Meinhardt model of clusters and spikes in clusters (slow activator fast inhibitor). Spotty - spiky solutions}

We now discuss briefly a continuous model that has some similarity to the Bak-Sneppen model. In the realization of the algorithm of Bak-Sneppen SOC for the convection - precipitation case we introduce: (1) the function $\mathrm{A}$ : activity at the location $(x, y) ;(2)$ the function $\mathrm{H}$ : degree of chances for instability, or: potential instability, which is the water vapor content. In any point, the "barrier" against an update is determined as the inverse of the content of water vapors, $=1 / \mathrm{H}$. These two functions $\mathrm{A}$ and $\mathrm{H}$ have a mutual relationship which is similar to the activator and inhibitor in the Gierer Meinhardt (GM) model (Meinhardt, 1982). The Bak-Sneppen model is discrete and algorthmic, the Gierer Meinhardt model is space and time continuous. There are elements that suggests the adoption of the GM model as a low-order, continuous limit of the ensemble of precipitation events. The GM model have spotty - spiky solutions which can be associated to extreme events.

The current approach to the to local convection dynamics is originated in the mass-flux parameterization with different developments (Yano, submitted). Assume there are centers of convection in the surface of a grid box. There is a parameter (called barrier) representing the degree of fitness of the local sub-system to the environment. For every point (sub - system) the value of that parameter is a random number. We examine the possible similarity 
by starting from the Bak-Sneppen dynamics. (1) The sub-system with the lowest value of the parameter (barrier) is found. This may be the point with the highest degree of Column Water Vapor content, since this means high chance for instability. A simplified representation is to consider the inverse of the amount of Column Water Vapor looks similar to

$$
\text { barrier } \sim \frac{1}{C W V} \sim \frac{1}{H}
$$

which appears on the Gierer Mainhardt model. The smallest barrier means highest chance to become unstable and then to be updated. The nonlinearity is the GM model is

$$
\frac{A^{2}}{H} \sim(\text { barrier }) \times A^{2}
$$

(2) The sub-system is mutated into a different state. A new barrier is atributted to this new sub-system. The new value of the barrier is taken at random from the same set $(0,1)$ with uniform probability. This is equivalent to assume that some precipitation has been produced and after that the Column Water Vapor is different in that specific sub-system. (3) Other sub-systems are affected by this mutation. They are also updated with the same occasion. The update is random, with new barriers from the set $(0,1)$. This may be interpreted as follows: other centers of convection has been influenced by the downdraft from the main sub-system. (4) The update of the connected species is annealed: the species that are chosen to be updated are chosen anew every time it changes. We can imagine that other centers of convection are affected by the update of the center that was active at the current moment.

The equations

$$
\begin{aligned}
\frac{\partial A}{\partial t} & =\varepsilon^{2} \Delta A-A+\frac{A^{2}}{H} \\
\tau \frac{\partial H}{\partial t} & =D \Delta H-H+A^{2}
\end{aligned}
$$

where $A, H>0$, with

$$
\begin{aligned}
& A \equiv \text { concentration of activator at point }(x, y, t) \\
& H \equiv \text { concentration of inhibitor at point }(x, y, t)
\end{aligned}
$$

The solutions show function activator $A$ concentrated in $K$ points, in different location of the domain $\Omega$. There is a phenomenon of solution concentration for $\varepsilon \rightarrow 0$ the peaks become more and more narrow and at the limit they are the points themselves. 
Looking for the comparison with the Bak - Sneppen dynamics, we have

$$
\begin{aligned}
\frac{\partial A}{\partial t} & =-A\left(1-\frac{A}{H}\right) \\
A & \sim \exp \left[-\left(1-\frac{A}{H}\right) t\right]
\end{aligned}
$$

and we comment as follows

$$
A \sim \exp [-t]
$$

if there would be no other term, i.e. no factor $\left(1-\frac{A}{H}\right)$. Then the time constant is

$$
A \sim \exp \left[-\frac{t}{\tau}\right] \text { with } \tau=1
$$

However we have

$$
\begin{aligned}
A & \sim \exp \left[-\left(1-\frac{A}{H}\right) t\right] \\
A & \sim \exp \left[-\frac{t}{\tau_{H}}\right] \\
\text { where } \tau_{H} & =\frac{1}{1-\frac{A}{H}}>\tau
\end{aligned}
$$

so the time of decay of the activator is longer when the nonlinear term is included. Assume for the moment that

$$
A / H<1
$$

If the $H$ function is very large, which means high content of vapor (large CWV) then $1-\frac{A}{H} \rightarrow 1$ and $\tau_{H} \searrow \tau$ and the decay of the local value of the activator $A$ is again fast. The large amount of vapor induces instability and favors the local reduction of the activator function.

Similarly we can evaluate qualitatively the role of the current value of the activator. If initially there is a high $A$ (but still $A / H<1$ ) then the time of decay $\tau_{H}$ is longer than $\tau$. The continuous decay of $A$ makes $1-A / H$ to approach 1 and the decay becomes faster, $\tau_{H} \searrow \tau$. This underlines the nonlinear effect of $A$ : High value of the activator are more persistent. The decay of the activator $A$ begins by having a slow rate but the rate accelerates in time.

Within the range where

$$
A / H<1
$$

the roles of large $A$ and $H$ are opposite: large initial $A$ tends to create persistence of $A$, but large $H$ induces instability and faster decay of $A$. 
Now, let us consider the range

$$
\frac{A}{H}>1
$$

This range can be reached by decreasing $H$ which means reducing the amount of vapor content. Then the activator $A$ increases exponentially. When $A$ grows and enters the regime $A / H>1$ the growth is self-accelerating since the coefficient of the exponent grows as $A$ itself.

Or, the regime can be obtained for higher $A$. The same self-acceleration occurs.

This leads to high concentration of the activator $A$ in few localised regions, where it happened that $A>H$ and growth with self-amplification has occured.

In the rest, the quantity of activator $A$ is smaller than the vapor content $H$ and there is quiet state, with no dynamics. $H$ may be seen as a passive inhibitor, in the sense that its presence means that there are chances for instability but the instability has not yet started.

If a mapping to the atmospheric convection is plausible we will name (a) the function $A$ is activity at the location $(x, y)$; and (2) the function $H$ is degree of chances for instability: potential instability. When the degree of chance of instability (potential instability) is larger than the local activity $(H>A)$ the activity has not yet started. The activity $A$ is low. The socalled inhibitor $H$ does NOT suppress the activity but is just a measure in which this one has not yet started.

When the potential instability is lower than the activity, $H<A$ this means that the activity has started in that point and the growth of the activity is very efficient.

Now, what is the connection between this system and the Bak - Sneppen model?

The Bak - Sneppen dynamics rises continuously the level of fitness, the value of the parameter that shows the degree of adaptation to the environment. This means that the points that have been updated are now more stable than they were immediately after the random initialization.

This however does not mean stability. It only means that the degree of stability (or fitness) has become rather similar for all the sub-systems of the system. There are no more large discrepancies that makes of the update a localised and isolated dynamics. Now a change can affect a large number of sub-systems and creates an avalanche. The system is in a critical state and it has reached that state by its own dynamics (is self - organized at criticality). 


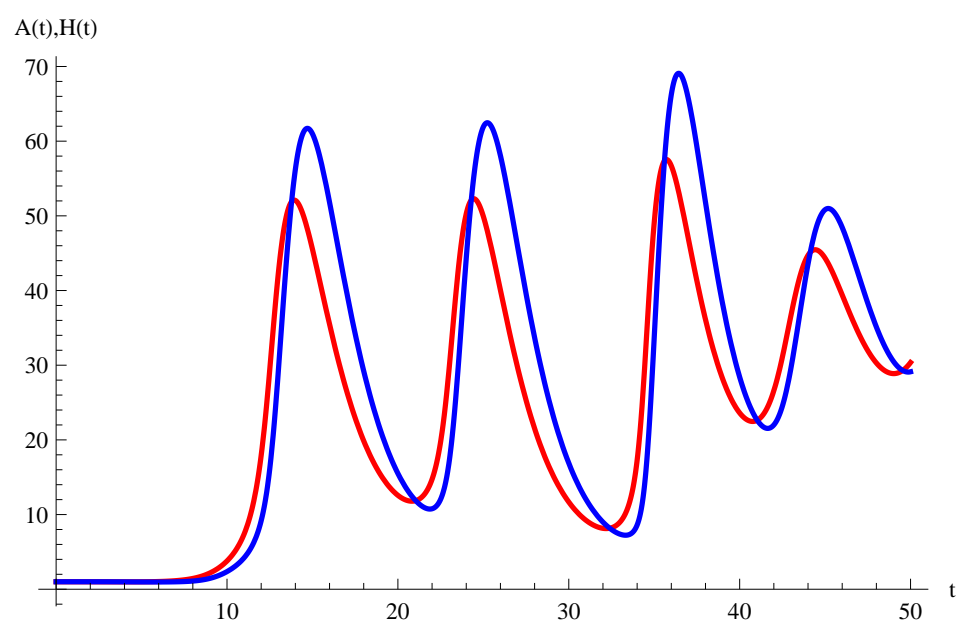

Figure 5: Time variation of the two functions $A(x, y)$ (activity) and $H(x, y)$ (column vapor water content) resulting from the $1 D$ Gierer-Meinhardt model. Oscillatory regime

The degree of fitness is the "barrier" of the sub-system against mutation. It is then the inverse of the amount of vapor,

$$
\begin{aligned}
& \text { "barrier" against mutation } \\
= & \text { fitness }=\frac{1}{C W V}=\frac{1}{H}
\end{aligned}
$$

is the inverse of the inhibitor. If there is large CWV (vapor) $H$ in a point then this means that there is low fitness, low "barrier" against mutation and the chances of instability are large. However when the vapor $H$ is measured there is no or low activity $A$ in that point. The operation of update consists of conversion of some vapor $H$ into activity $A$ : the vapor $H$ decreases and the activity $A$ increases, the barrier is increased as required by the Bak Sneppen dynamics. The activity $A$ may increase but not sufficiently such that $A>H$. Then what we get is just another landscape of $A(x, y)$ and $H(x, y)$ but not spiky solution.

After the update we have a smaller $H$ (less vapors) in the updated locations, which means higher barriers. The activity has also increased to a certain extent in those points.

The standard Bak - Sneppen random dynamics has been realised by a random factor of conversion from the vapor content $H$ to the activity $A$.

After a sequence of such updates the activity $A$ will be higher than initially, and with all points being at comparable degrees of activity. In the same time, the vapor has decreased in almost all points (i.e. the barriers have increased for those points). 


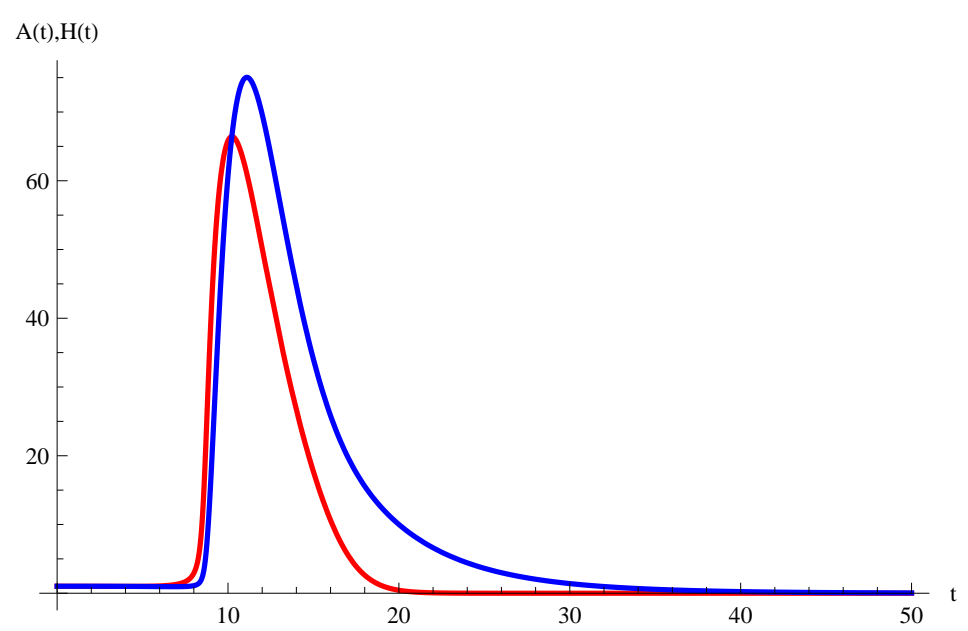

Figure 6: Same as Figure (5). A regime of fast compensation and decay.

\subsection{Spikes in the solutions of the Gierer - Mainhardt model}

The system GM in $2 D$, for $t>0$ and boundary conditions

$$
\partial_{n} A=0, \partial_{n} H=0 \text { for } x \in \partial \Omega
$$

exhibits spike solution that will tend to $\infty$ for $\varepsilon \rightarrow 0$. We have solved these equations and indeed we have found that there is concentration of the activity in localizes areas where it can have large amplitudes. This hsould require a more detailed understanding of the

\section{Conclusions}

The atmospheric convection has many manifestations and a unique description of the specific regimes is difficult. However there are situations where the atmospheric convection can be seen as a part of the complex phenomenon with the essential characteristics of the self-organized criticality: the slow driving, the fast reaction when there is departure from quasi-equilibrium, the interaction between neighbor sites and the formation of large-scale ensembles with correlated response. The correlations of fluctuations of SOC systems should exhibit universal scaling in space and time and this is indeed found for the SOC regimes of convection. Observational data are compatible with the results of analytical derivation based on a paradigmatic example of punctuated equilibrium system, the Bak-Sneppen model. We provide detail 


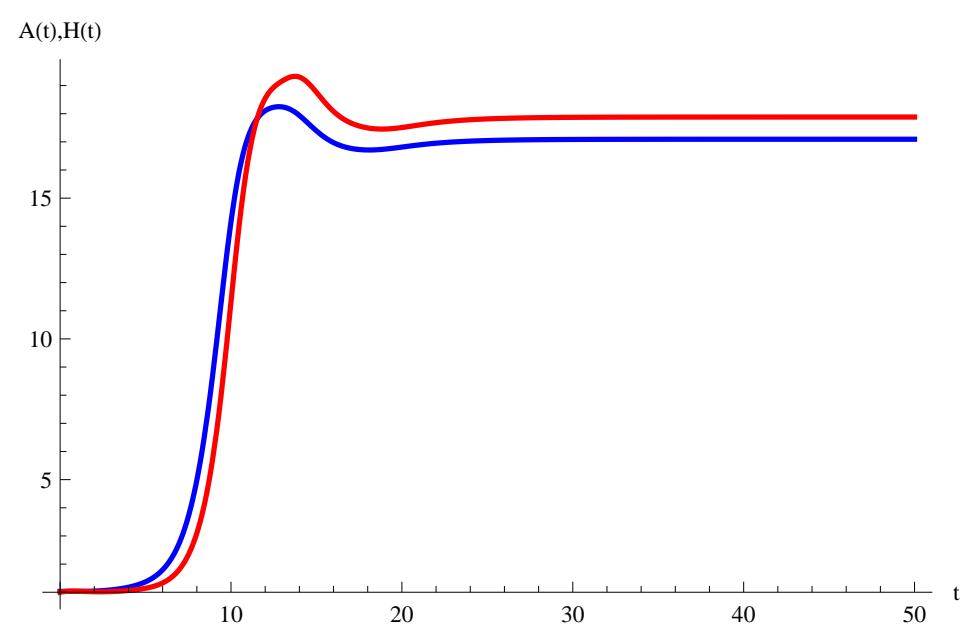

Figure 7: Same as Figure (5). A regime of saturation.

analytical derivation of the equations for the probabilities of states of the sequential update of the Bak-Sneppen model with $k=2$ random neighbors.

We present a possible extension to continuum dynamics, by the model Gierer-Meinhardt. The numerical investigation confirms, to a certain extent, the expectation: the system evolves by a continuous mutual control of an inhibitor and an activator variables. It also presents spiky solutions that may be of interest in the investigation of the extreme dynamics.

In conclusion, SOC appears to be a possible ground for construction of a coherent perspective on the large scale organization of convection.

Acknowledgments. This work has been partly supported by the grant ERC-Like nr.4/2012 of UEFISCDI Romania. The exchange of ideas in the COST ES0905 collaboration is particularly acknowledged.

\section{Appendix.Calculation of the probabilities of the states for Bak-Sneppen dynamics at $K=2$ random neighbor}

\subsection{Definition and calculation of the transition proba- bilities}

Exact analytic results exist for the time-dependent statistical characteristics of the Bak - Sneppen model. These have been obtained by de Boer et al. 
(1995), de Boer et al. (1994), Paczuski et al. (1996). In this Appendix we start from these original works and provide details of the calculations leading to the system of equation connecting the probabilities of the states before and after update.

The case that will be examined has $K=2$. This means that besides the sites with the smallest value $x_{i}$ only just another one $x_{l}$ is changed by replacing $x_{l}$ with a new $x_{l}^{\prime}$, random, value. The site $x_{l}$ is chosen at random.

We fix a real value for the parameter $\lambda$. Consider the number $n$ of sites that have the value $x_{i}$ less than $\lambda$. Define

$$
P_{n}(t)
$$

as the probability that at time $t$ there are $n$ sites that have value $x_{i}$ lower than $\lambda$.

This probability verifies the following master equation

$$
P_{n}(t+1)=\sum_{m=0}^{N} M_{n, m} P_{m}(t)
$$

where

$$
\begin{aligned}
& M_{n+1, n}= \lambda^{2}-\lambda^{2} \frac{n-1}{N-1} \\
& M_{n, n}= 2 \lambda(1-\lambda)+\left(3 \lambda^{2}-2 \lambda\right) \frac{n-1}{N-1} \\
& M_{n-1, n}=(1-\lambda)^{2}+\left(-3 \lambda^{2}+4 \lambda-1\right) \frac{n-1}{N-1} \\
& M_{n-2, n}=(1-\lambda)^{2} \frac{n-1}{N-1} \\
& M_{0,0}=(1-\lambda)^{2} \\
& M_{1,0}=2 \lambda(1-\lambda) \\
& M_{2,0}=\lambda^{2}
\end{aligned}
$$

It is assumed that the total number of sites in the system is $N$. At time $t$ there are $m$ sites for which $x_{j}<\lambda$. The probability that at time $t$ there are $m$ such sites is $P_{m}(t)$.

Now we apply the algorithmic change at step $t$.

Identify the site $k$ with the lowest value $x_{k}$ from the set of $m$ sites. This is made with probability $1 / m$ because any of the $m$ sites can be at this state.

$$
\text { probability that the site } k \text { has } x_{k} \text { lower than } \lambda \text { is } \frac{1}{m}
$$


Replace $x_{k}$ by the result $x_{k}^{\prime}$ of extracting a random number from $[0,1]$ with uniform probability

$$
\text { site } k: x_{k} \rightarrow x_{k}^{\prime}
$$

Now we examine the two possibilities.

A. There is a chance that the new $x_{k}^{\prime}$ value is larger than $\lambda$

probability that the site $k$ with minimum $x_{k}$ (principal) is updat(684) to a value $x_{k}^{\prime}$ which is greater than $\lambda$

$=1-\lambda$

since the distribution is uniform on $[0,1]$ and the probability is given by the length of the interval. In this case the number of sites with value $x$ smaller than $\lambda$ at the next time step $t+1$ is smaller by one unit

$$
m \rightarrow m-1
$$

Simultaneously we have to identify a site $l$ that is in interaction with the site $k$. This is done under the assumption that this other site is any of the available $N-1$ sites remaining, at random, with uniform probability. Therefore we would be tempted to say that the probability of choosing the site $l$ from the rest of $N-1$ sites is $1 /(N-1)$.

However we must distinguish between the possibilities that $l$ has its $x_{l}$ smaller or larger than $\lambda$. Then we divide this step into two branches:

\section{A.1 Assume that}

$$
\text { secondary } x_{l}<\lambda
$$

Then it is one of the $m$ sites that are all characterized at $t$ as having $x_{i}<\lambda$. (To this family belongs also the main site, the lowest, $x_{k}$ ).

The probability for this is

$$
\begin{aligned}
& \frac{m-1}{N-1} \text { (probability to choose } l \text { between the } m-1 \text { sites with } x_{i}<l \beta 7 \text { ) } \\
& m-1 \text { sites are remaining after the site } x_{k} \text { is special, the lowest }
\end{aligned}
$$

Then we have two possible evolutions:

A.1.1 The update of the site $l$ is such that the new value of $x_{l}$ is larger than $\lambda$.

$$
x_{l} \rightarrow x_{l}^{\prime}>\lambda
$$


with

probability that the secondary site $l$ belongs to the set $m-1$ and(39) after update $x_{l}^{\prime}$ is grater than $\lambda$ is

$$
\frac{m-1}{N-1} \times(1-\lambda)
$$

This update of the other (interacting) site reduces the number of sites in the initial set $m$ with one unit. This reduction comes after the first reduction with one unit, made by the the principal site, $x_{k}$, the lowest at $t$, which we assumed has been updated to $x_{k}^{\prime}>\lambda$.

Assume that we have $m$ sites that are initially under $\lambda$. Due to these two updates, the number $m$ of sites having $x<\lambda$ changes as

$$
\begin{aligned}
x_{k} & \rightarrow x_{k}^{\prime}: m \rightarrow m-1 \quad(\text { the main site escapes to }>\lambda) \\
x_{l} & \rightarrow x_{l}^{\prime}: m-1 \rightarrow m-2 \quad(\text { the secondary site escapes to }>\lambda)
\end{aligned}
$$

the number of sites with $x^{\prime} s$ less than $\lambda$ is

number of sites with $x$ less than $\lambda$, at time $t+1$ is(41)

$$
(m-1)-1=m-2
$$

The transition is

$$
P_{m}(t) \rightarrow P_{m-2}(t+1)
$$

which means a contribution to

$$
P_{m-2}(t+1)
$$

and the contribution is

$$
\begin{aligned}
& (1-\lambda) \times \frac{m-1}{N-1} \times(1-\lambda) \\
= & \frac{m-1}{N-1}(1-\lambda)^{2}
\end{aligned}
$$

A.1.2. The update of the secondary (interacting) site $l$ is such that the new value of $x_{l}$ is smaller than $\lambda$.

$$
x_{l} \rightarrow x_{l}^{\prime}<\lambda
$$

with

probability that the secondary site $l$ belongs to the set $m-1$ an $(46)$ after update $x_{l}^{\prime}$ is less than $\lambda$ is $\frac{m-1}{N-1} \times \lambda$ 
which maintains the number of sites in the initial set $m$. Due to these two updates

$x_{k} \rightarrow x_{k}^{\prime}: m \rightarrow m-1 \quad$ (the lowest site escapes to $>\lambda$ )

$x_{l} \rightarrow x_{l}^{\prime}: m-1 \rightarrow m-1 \quad\left(\begin{array}{c}\text { the secondary site is not from the } m \text { family } \\ \text { and remains ouside this family }\end{array}\right)$

the number of sites with $x^{\prime} s$ less than $\lambda$ is

number of sites with $x$ less than $\lambda$, at time $t+1$ is

$m-1$

The transition represented by these updates is

$$
P_{m}(t) \rightarrow P_{m-1}(t+1)
$$

which means a contribution to

$$
P_{m-1}(t+1)
$$

and the contribution is

$$
\begin{aligned}
& (1-\lambda) \times \frac{m-1}{N-1} \times \lambda \\
= & \frac{m-1}{N-1}(1-\lambda) \lambda
\end{aligned}
$$

A.2. Now assume that the secondary, interacting, site $x_{l}$ has at $t$ a barrier which is higher than the limit $\lambda$,

$$
\text { secondary } x_{l}>\lambda
$$

Then it is one of the $N-m$ sites that are all characterized at $t$ as having $x_{i}>\lambda$.

The probability for this is

$$
\frac{N-m}{N-1} \text { (probability to choose } l \text { between the } N-m \text { sites with } x_{i}>l \text { at } t \text { ) }
$$

Then we have two possible evolutions:

A.2.1 The update of the (interacting) site $l$ (which belonged to $N-m$ ) is such that the new value of $x_{l}$ is larger than $\lambda$.

$$
x_{l} \rightarrow x_{l}^{\prime}>\lambda
$$


with

probability that the secondary site $l$ belongs to the set $N-m$ an $(55)$ after update $x_{l}^{\prime}$ is grater than $\lambda$ is

$\frac{N-m}{N-1} \times(1-\lambda)$

which keeps constant the number of sites in the initial set $m$. Due to these two updates

$x_{k} \rightarrow x_{k}^{\prime}: m \rightarrow m-1$ (the lowest site escapes to higher $\lambda$ )

$x_{l} \rightarrow x_{l}^{\prime}: m-1 \rightarrow m-1\left(\begin{array}{c}\text { the secondary site does not belong to } m-1 \\ \text { and it does not enter under } \lambda\end{array}\right)$

the number of sites with $x^{\prime} s$ less than $\lambda$ is

number of sites with $x$ less than $\lambda$, at time $t+1$ is

$m-1$

which means a transition

$$
P_{m}(t) \rightarrow P_{m-1}(t+1)
$$

through a contribution to

$$
P_{m-1}(t+1)
$$

and the contribution is

$$
\begin{aligned}
& (1-\lambda) \times \frac{N-m}{N-1} \times(1-\lambda) \\
= & \frac{N-m}{N-1}(1-\lambda)^{2}
\end{aligned}
$$

A.2.2. The update of the (interacting) site $l$ (which belonged to $N-m$, having $>\lambda$ ) is such that the new value of $x_{l}$ is smaller than $\lambda$.

$$
x_{l} \rightarrow x_{l}^{\prime}<\lambda
$$

with

probability that the secondary site $l$ belongs to the set $N-m$ and after update $x_{l}^{\prime}$ is less than $\lambda$ is

$\frac{N-m}{N-1} \times \lambda$ 
which increases the number of sites in the initial set $m$ with one unit. Due to these two updates

$$
\begin{aligned}
& \left.x_{k} \rightarrow x_{k}^{\prime}: m \rightarrow m-1 \text { (the main site escapes to }>\lambda\right) \\
& x_{l} \rightarrow x_{l}^{\prime}: m-1 \rightarrow m-1+1\left(\begin{array}{c}
\text { the secondary site did not belong to } m \text { family } \\
\text { but now comes under } \lambda
\end{array}\right)
\end{aligned}
$$

the number of sites with $x^{\prime} s$ less than $\lambda$ is

$$
\begin{aligned}
& \text { number of sites with } x \text { less than } \lambda \text {, at time } t+1 \text { is } \\
& m
\end{aligned}
$$

[one leaves $\left(x_{k}\right)$ and other comes $\left.\left(x_{l}\right)\right]$.

This case connects the situations

$$
P_{m}(t) \rightarrow P_{m}(t+1)
$$

which means a contribution to

$$
P_{m}(t+1)
$$

and the contribution is

$$
\begin{aligned}
& (1-\lambda) \times \frac{N-m}{N-1} \times \lambda \\
= & \frac{N-m}{N-1}(1-\lambda) \lambda
\end{aligned}
$$

B. There is a chance that the new $x_{k}^{\prime}$ value is smaller than $\lambda$

probability that the site $k$ with minimum $x_{k}$ (principal) is updated to a value $x_{k}^{\prime}$ which is smaller than $\lambda$

$$
=\lambda
$$

In this case the number of sites with value $x$ smaller than $\lambda$ at the next time step $t+1$ does not change

$$
m \rightarrow m
$$

Simultaneously we have to identify a site $l$ that is in interaction with the site $k$. This is done under the assumption that this other site is any of the available $N-1$ sites remaining. Therefore we would be tempted to say that the probability of choosing the site $l$ from the rest of $N-1$ sites is $1 /(N-1)$. 
However we must distinguish between the possibilities that $l$ has its $x_{l}$ smaller or larger than $\lambda$. Then we divide this step into two branches:

B.1 Assume that

$$
\text { secondary (interacting) site } x_{l}<\lambda
$$

Then it is one of the $m$ sites that are all characterized at $t$ as having $x_{i}<\lambda$. The probability for this is

$$
\frac{m-1}{N-1} \text { (probability to choose } l \text { between the } m \text { sites with } x_{i}<l \text { ) }
$$

According to what happens with $x_{l}$ after update, we have two possible evolutions:

B.1.1 The update of the site $l$ is such that the new value of $x_{l}$ is larger than $\lambda$.

$$
x_{l} \rightarrow x_{l}^{\prime}>\lambda
$$

with

probability that the secondary site $l$ belongs to the set $m$ and after update $x_{l}^{\prime}$ is grater than $\lambda$ is

$$
\frac{m-1}{N-1} \times(1-\lambda)
$$

which reduces the number of sites in the initial set $m$ with one unit. Due to these two updates

$$
\begin{aligned}
& \left.x_{k} \rightarrow x_{k}^{\prime}: m \rightarrow m \text { (the main site remains under } \lambda\right) \\
& x_{l} \rightarrow x_{l}^{\prime}: m \rightarrow m-1\left(\begin{array}{c}
\text { the secondary site was in the } m \text { family } \\
\text { but now escapes to }>\lambda
\end{array}\right)
\end{aligned}
$$

the number of sites with $x^{\prime} s$ less than $\lambda$ is

$$
\begin{aligned}
& \text { number of sites with } x \text { less than } \lambda \text {, at time } t+1 \text { is } \\
& m-1
\end{aligned}
$$

The transition is the following

$$
P_{m}(t) \rightarrow P_{m-1}(t+1)
$$

which means a contribution to

$$
P_{m-1}(t+1)
$$


and the contribution is to $M_{m-1, m}$.

$$
\begin{aligned}
& \lambda \times \frac{m-1}{N-1} \times(1-\lambda) \\
= & \frac{m-1}{N-1}(1-\lambda) \lambda
\end{aligned}
$$

B.1.2. The update of the site $l$ is such that the new value of $x_{l}$ is smaller than $\lambda$.

$$
x_{l} \rightarrow x_{l}^{\prime}<\lambda
$$

with

probability that the secondary site $l$ belongs to the set $m$ and after update $x_{l}^{\prime}$ is less than $\lambda$ is

$$
\frac{m-1}{N-1} \times \lambda
$$

which maintains the number of sites in the initial set $m$. Due to these two updates

$$
\begin{aligned}
& x_{k} \rightarrow x_{k}^{\prime}: m \rightarrow m \quad(\text { the main site remains in } m) \\
& x_{l} \rightarrow x_{l}^{\prime}: m \rightarrow m(\text { the secondary site remains within } m)
\end{aligned}
$$

the number of sites with $x^{\prime} s$ less than $\lambda$ is

$$
\begin{aligned}
& \text { number of sites with } x \text { less than } \lambda \text {, at time } t+1 \text { is } \\
& m
\end{aligned}
$$

The transition is

$$
P_{m}(t) \rightarrow P_{m}(t+1)
$$

which means a contribution to

$$
P_{m}(t+1)
$$

and the contribution is to $M_{m, m}$.

$$
\begin{aligned}
& \lambda \times \frac{m-1}{N-1} \times \lambda \\
= & \frac{m-1}{N-1} \lambda^{2}
\end{aligned}
$$


B.2. Assume that

secondary, interacting, site $x_{l}>\lambda$

Then it is one of the $N-m$ sites that are all characterized at $t$ as having $x_{i}>\lambda$.

The probability for this is

$\frac{N-m}{N-1}$ (probability to choose $l$ between the $N-m$ sites with $x_{i}>l$ at $t$ )

Then we have two possible evolutions:

B.2.1 The update of the site $l$ (which belonged to $N-m$ ) is such that the new value of $x_{l}$ is larger than $\lambda$.

$$
x_{l} \rightarrow x_{l}^{\prime}>\lambda
$$

with

probability that the secondary site $l$ belongs to the set $N-m$ and after update $x_{l}^{\prime}$ is grater than $\lambda$ is

$\frac{N-m}{N-1} \times(1-\lambda)$

which keeps constant the number of sites in the initial set $m$. Due to these two updates

$$
\begin{gathered}
x_{k} \rightarrow x_{k}^{\prime}: m \rightarrow m \\
x_{l} \rightarrow x_{l}^{\prime}: m \rightarrow m
\end{gathered}
$$

the number of sites with $x^{\prime} s$ less than $\lambda$ is

$$
\begin{aligned}
& \text { number of sites with } x \text { less than } \lambda \text {, at time } t+1 \text { is } \\
& m
\end{aligned}
$$

The transition is

$$
P_{m}(t) \rightarrow P_{m}(t+1)
$$

which means a contribution to

$$
P_{m}(t+1)
$$


and the contribution is to $M_{m, m}$.

$$
\begin{aligned}
& \lambda \times \frac{N-m}{N-1} \times(1-\lambda) \\
= & \frac{N-m}{N-1}(1-\lambda) \lambda
\end{aligned}
$$

B.2.2. As assumed in the class B.2 the secondary, interacting site has the barrier $>\lambda$, which means that it does not belong to the family $m$ like $x_{k}$, but to $N-m$. The update of the site $l$ (which belonged to $N-m$ ) is such that the new value of $x_{l}$ is smaller than $\lambda$.

$$
x_{l} \rightarrow x_{l}^{\prime}<\lambda
$$

with

probability that the secondary site $l$ belongs to the set $N-m$ and after update $x_{l}^{\prime}$ is less than $\lambda$ is

$$
\frac{N-m}{N-1} \times \lambda
$$

which increases the number of sites in the initial set $m$ with one unit. Due to these two updates

$x_{k} \rightarrow x_{k}^{\prime}: m \rightarrow m$ (the main site stays inside $m$ family)

$x_{l} \rightarrow x_{l}^{\prime}: m \rightarrow m+1 \quad$ (the secondary site, initially out, comes to the $m$ family)

the number of sites with $x^{\prime} s$ less than $\lambda$ is

$$
\begin{aligned}
& \text { number of sites with } x \text { less than } \lambda \text {, at time } t+1 \text { is } \\
& m+1
\end{aligned}
$$

The transition is

$$
P_{m}(t) \rightarrow P_{m+1}(t+1)
$$

which means a contribution to

$$
P_{m+1}(t+1)
$$

and the contribution is to $M_{m+1, m}$.

$$
\begin{aligned}
& \lambda \times \frac{N-m}{N-1} \times \lambda \\
= & \frac{N-m}{N-1} \lambda^{2}
\end{aligned}
$$




\section{$7.2 \quad$ Results}

Now let us collect the results, i.e. write the expression of the elements of the matrix of transition $M_{n, m}$. They connect the state $m$ at time $t$ (whose probability is $\left.P_{m}(t)\right)$ with the state $n$ at time $t+1$, whose probability is $P_{n}(t+1)$. We have seen that the transitions having $n$ as final state can only originate from states of the small set $n, n \pm 1, n-2$. Then we can replace the generic notation $m$ with the appropriate value from this set. The Table below lists the connections that are possible as transitions.

\begin{tabular}{|llllll|}
\hline & $A 1 . x_{l}<\lambda$ & $A 1.1$ & $x_{l}^{\prime}>\lambda$ & $\frac{n-1}{N-1}(1-\lambda)^{2}$ & $n-2$ \\
A. $x_{k}^{\prime}>\lambda$ & & $A 1.2$ & $x_{l}^{\prime}<\lambda$ & $\frac{n-1}{N-1} \lambda(1-\lambda)$ & $n-1$ \\
& $A 2 . x_{l}>\lambda$ & $A 2.1$ & $x_{l}^{\prime}>\lambda$ & $\frac{N-n}{N-1}(1-\lambda)^{2}$ & $n-1$ \\
& & $A 2.2$ & $x_{l}^{\prime}<\lambda$ & $\frac{N-n}{N-1} \lambda(1-\lambda)$ & $n$ \\
\hline \multirow{5}{*}{$B . x_{k}^{\prime}<\lambda$} & \multirow{2}{*}{$B 1 . x_{l}<\lambda$} & $B 1.1$ & $x_{l}^{\prime}>\lambda$ & $\frac{n-1}{N-1} \lambda(1-\lambda)$ & $n-1$ \\
& & $B 1.2$ & $x_{l}^{\prime}<\lambda$ & $\frac{n-1}{N-1} \lambda^{2}$ & $n$ \\
& $B 2 . x_{l}>\lambda$ & $B 2.1$ & $x_{l}^{\prime}>\lambda$ & $\frac{N-n}{N-1} \lambda(1-\lambda)$ & $n$ \\
& & $B 2.2$ & $x_{l}^{\prime}<\lambda$ & $\frac{N-n}{N-1} \lambda^{2}$ & $n+1$ \\
\hline
\end{tabular}

\subsection{Calculation of the elements of the transition ma- trix}

Now we use this Table to produce the transition probabilities $M_{n, m}$, with $m$ from the small set. With the destination state $n$, we add the contributions that originate in one or several states of the set.

\subsubsection{The case $M_{n-2, n}$}

We take the single case of this type

$$
P_{n}(t) \rightarrow P_{n-2}(t+1)
$$

which is made by

$$
\text { A.1.1 }
$$

with the probability

$$
M_{n-2, n}=\frac{n-1}{N-1}(1-\lambda)^{2}
$$




\subsubsection{The case $M_{n, n}$}

We have to put together the cases where

- we start from $n$ sites with less than $\lambda$, and

- we arrive at $n$ sites less than $\lambda$

The transition is

$$
P_{n}(t) \rightarrow P_{n}(t+1)
$$

The sum is

$$
\begin{gathered}
A .2 .2 \rightarrow \frac{N-n}{N-1} \lambda(1-\lambda) \\
B .1 .2 \rightarrow \frac{n-1}{N-1} \lambda^{2} \\
B .2 .1 \quad \rightarrow \quad \frac{N-n}{N-1}(1-\lambda) \lambda \\
=\frac{N-1-n+1}{N-1} 2 \lambda(1-\lambda)+\frac{n-1}{N-1} \lambda^{2} \\
=2 \lambda(1-\lambda)+\frac{1}{N-1}\left[-(n-1) 2 \lambda(1-\lambda)+(n-1) \lambda^{2}\right] \\
=2 \lambda(1-\lambda)+\frac{1}{N-1}(n-1)\left[-2 \lambda+2 \lambda^{2}+\lambda^{2}\right] \\
=2 \lambda(1-\lambda)+\frac{1}{N-1}(n-1)\left(3 \lambda^{2}-2 \lambda\right)
\end{gathered}
$$

This is $P_{n, n}$ and is

$$
M_{n, n}=2 \lambda(1-\lambda)+\frac{1}{N-1}(n-1)\left(3 \lambda^{2}-2 \lambda\right)
$$

and is $\mathrm{OK}$.

\subsubsection{The case $M_{n-1, n}$}

We have to put together the cases where

- we start from $n$ sites with less than $\lambda$, and

- we arrive at $n-1$ sites less than $\lambda$ 
The transition is

$$
P_{n}(t) \rightarrow P_{n-1}(t+1)
$$

The sum is

$$
\begin{gathered}
A .1 .2 \rightarrow \frac{n-1}{N-1} \lambda(1-\lambda) \\
A .2 .1 \rightarrow \frac{N-n}{N-1}(1-\lambda)^{2} \\
B .1 .1 \rightarrow \frac{n-1}{N-1} \lambda(1-\lambda) \\
\quad \frac{N-n}{N-1}(1-\lambda)^{2}+2 \frac{n-1}{N-1} \lambda(1-\lambda) \\
=\frac{N-1-n+1}{N-1}(1-\lambda)^{2}+2 \frac{n-1}{N-1} \lambda(1-\lambda) \\
=\quad(1-\lambda)^{2}+\frac{n-1}{N-1}\left[-(1-\lambda)^{2}+2 \lambda(1-\lambda)\right] \\
=(1-\lambda)^{2}+\frac{n-1}{N-1}\left(-1+2 \lambda-\lambda^{2}+2 \lambda-2 \lambda^{2}\right) \\
=(1-\lambda)^{2}+\frac{n-1}{N-1}\left(-1+4 \lambda-3 \lambda^{2}\right)
\end{gathered}
$$

This is $M_{n-1, n}$ and is

$$
M_{n-1, n}=(1-\lambda)^{2}+\frac{n-1}{N-1}\left(-1+4 \lambda-3 \lambda^{2}\right)
$$

and is OK.

\subsubsection{The case $M_{n+1, n}$}

We find the situation

$$
\text { B. } 2.2
$$

with the probability

$$
\begin{aligned}
M_{n+1, n} & =\frac{N-n}{N-1} \lambda^{2} \\
& =\frac{N-1-n+1}{N-1} \lambda^{2}=\lambda^{2}-\lambda^{2} \frac{n-1}{N-1}
\end{aligned}
$$

and is OK.

These are cases for

$$
n \geq 1
$$




\subsubsection{The particular case $M_{0,0}$}

This corresponds to the following situation: at time $t$ there is no site under $\lambda$. At time $t+1$ the number of sites under $\lambda$ is not modified, it is zero. This means that the update of $x_{k}$, the minimum site, takes it from $>\lambda$ and keeps it somewhere $>\lambda$. For the secondary, interacting, site, it was initially $>\lambda$ and after update it remains $>\lambda$. The probability of this transition is the product of two probabilities

- the probability that $x_{k}$ takes after update a value that is greater than $\lambda$,

$$
1-\lambda
$$

- the probability that $x_{l}$ takes after update a value that is greater than $\lambda$,

$$
1-\lambda
$$

The product is the element of matrix

$$
M_{0,0}=(1-\lambda)^{2}
$$

\subsubsection{The particular case $M_{1,0}$}

This can be obtained in two ways:

- the lowest site $x_{k}$, which, - since we start from $m=0$, has at time $t$ the value $>\lambda$, is updated to the same region, with probability $(1-\lambda)$. In the same time the secondary site that initially at time $t$ is $>\lambda$ (since $m=0$ ) after update at time $t+1$ takes a value $<\lambda$, with probability $\lambda$. Then the contribution of this situation to the matrix element $M_{1,0}$ is

$$
M_{1,0}^{(1)}=\lambda(1-\lambda)
$$

- the lowest site $x_{k}$, which, - since we start from $m=0$, has at time $t$ the value $>\lambda$, is updated to the region that is under $\lambda$, with a probability $\lambda$. The secondary site $x_{l}$ is initially (at $\left.t\right)>\lambda$ and is updated remaining in this region $>\lambda$, with the probability $(1-\lambda)$. The contribution to the matrix element $M_{1,0}$ is

$$
M_{1,0}^{(2)}=\lambda(1-\lambda)
$$

Finally we get the total matrix element

$$
M_{1,0}=2 \lambda(1-\lambda)
$$




\subsubsection{The particular case $M_{2,0}$}

It is easy to see that

$$
M_{2,0}=\lambda^{2}
$$

This determines completely the set of transition probabilities (matrix elements $\left.M_{j, k}\right)$.

\subsection{Equations connecting the probabilities of the states at the update transition}

The knowledge of the elements of the transition matrix allows to write in detail the equations connecting the probabilties.

$$
P_{n}(t+1)=\sum_{m=0}^{N} M_{n, m} P_{m}(t)
$$

To use the results obtained above, we start with the lowest cases

$$
\begin{aligned}
& P_{0}(t+1)=M_{0,0} P_{0}(t)+M_{0,1} P_{1}(t)+M_{0,2} P_{2}(t) \\
& P_{1}(t+1)=M_{1,0} P_{0}(t)+M_{1,1} P_{1}(t)+M_{1,2} P_{2}(t) \\
& P_{2}(t+1)=M_{2,0} P_{0}(t)+M_{2,1} P_{1}(t)+M_{2,2} P_{2}(t)+M_{2,3} P_{3}(t)
\end{aligned}
$$

First equation

$$
\begin{aligned}
P_{0}(t+1)= & M_{0,0} P_{0}(t)+M_{0,1} P_{1}(t)+M_{0,2} P_{2}(t) \\
= & (1-\lambda)^{2} P_{0}(t) \\
& +\left[(1-\lambda)^{2}+\frac{n-1}{N-1}\left(-1+4 \lambda-3 \lambda^{2}\right)\right]_{n=1} P_{1}(t) \\
& +\left[\frac{n-1}{N-1}(1-\lambda)^{2}\right]_{n=1} P_{2}(t)
\end{aligned}
$$

and we take the limit $N \rightarrow \infty$,

$$
P_{0}(t+1)=(1-\lambda)^{2}\left[P_{0}(t)+P_{1}(t)\right]
$$

The second equation

$$
\begin{aligned}
P_{1}(t+1)= & M_{1,0} P_{0}(t)+M_{1,1} P_{1}(t)+M_{1,2} P_{2}(t) \\
= & 2 \lambda(1-\lambda) P_{0}(t) \\
& +\left[2 \lambda(1-\lambda)+\frac{1}{N-1}(n-1)\left(3 \lambda^{2}-2 \lambda\right)\right]_{n=1} P_{1}(t) \\
& +\left[(1-\lambda)^{2}+\frac{n-1}{N-1}\left(-1+4 \lambda-3 \lambda^{2}\right)\right]_{n=2} P_{2}(t)
\end{aligned}
$$




$$
\begin{aligned}
P_{1}(t+1)= & 2 \lambda(1-\lambda)\left[P_{0}(t)+P_{1}(t)\right] \\
& +\left[(1-\lambda)^{2}+\frac{1}{N-1}\left(-1+4 \lambda-3 \lambda^{2}\right)\right] P_{2}(t)
\end{aligned}
$$

at the limit

$$
N \rightarrow \infty
$$

the last term vanishes and we obtain

$$
P_{1}(t+1)=2 \lambda(1-\lambda)\left[P_{0}(t)+P_{1}(t)\right]+(1-\lambda)^{2} P_{2}(t)
$$

The third equation is

$$
\begin{aligned}
P_{2}(t+1)= & M_{2,0} P_{0}(t)+M_{2,1} P_{1}(t)+M_{2,2} P_{2}(t)+M_{2,3} P_{3}(t) \\
= & \lambda^{2} P_{0}(t) \\
& +\left[\lambda^{2}-\lambda^{2} \frac{n-1}{N-1}\right]_{n=1} P_{1}(t) \\
& +\left[2 \lambda(1-\lambda)+\frac{1}{N-1}(n-1)\left(3 \lambda^{2}-2 \lambda\right)\right]_{n=2} P_{2}(t) \\
& +\left[(1-\lambda)^{2}+\frac{n-1}{N-1}\left(-1+4 \lambda-3 \lambda^{2}\right)\right]_{n=3} P_{3}(t) \\
& P_{2}(t+1) \\
= & \lambda^{2} P_{0}(t)+\lambda^{2} P_{1}(t) \\
& +\left[2 \lambda(1-\lambda)+\frac{1}{N-1}\left(3 \lambda^{2}-2 \lambda\right)\right] P_{2}(t) \\
& +\left[(1-\lambda)^{2}+\frac{2}{N-1}\left(-1+4 \lambda-3 \lambda^{2}\right)\right] P_{3}(t)
\end{aligned}
$$

When

$$
N \rightarrow \infty
$$

the last terms in the paranthesis vanish

$$
\begin{aligned}
& P_{2}(t+1) \\
= & \lambda^{2}\left[P_{0}(t)+P_{1}(t)\right] \\
& +2 \lambda(1-\lambda) P_{2}(t) \\
& +(1-\lambda)^{2} P_{3}(t)
\end{aligned}
$$

The equation for

$$
n \geq 3
$$


In order to write this equation we have to count the transitions that are possible when we only have $K$ sites involved in updates. The first update is the lowest $x_{i}$ and the second is the interacting site $x_{l}$.

To reach $n$ at $t+1$ the following possibilities exist

$$
\begin{array}{rll}
\text { start from } n-1 & : & M_{n, n-1} \\
\text { start from } n & : & M_{n, n} \\
\text { start from } n+1 & : & M_{n, n+1}
\end{array}
$$

There is another possibility, to start from $n+2$ and consider the update of the main site $x_{k}$ that removes it from $<\lambda$ and places it above; and update of the interacting site $x_{l}$ takes it from $<\lambda$ and places it to $>\lambda$. This means reduction of the $n+2$ sites under $\lambda$ to $n$ under $\lambda$. The transition matrix is

$$
M_{n, n+2}
$$

which is obtained from

$$
M_{m-2, m}=\frac{m-1}{N-1}(1-\lambda)^{2}
$$

taking

$$
m=n+2
$$

which means

$$
M_{n, n+2}=\frac{n+1}{N-1}(1-\lambda)^{2}
$$

The limit $N \rightarrow \infty$ makes this transition element to vanish.

Then

$$
P_{n}(t+1)=M_{n, n-1} P_{n-1}(t)+M_{n, n} P_{n}(t)+M_{n, n+1} P_{n+1}(t)
$$

We have to adapt the transition matrix elements to the limit $N \rightarrow \infty$.

The probability of transition $M_{n, n-1}$ must be calculated from

$$
M_{n+1, n}=\lambda^{2}-\lambda^{2} \frac{n-1}{N-1}
$$

by the replacement $n \rightarrow n-1$,

$$
M_{n, n-1}=\lambda^{2}-\lambda^{2} \frac{n-2}{N-1}
$$

by taking the limit $N \rightarrow \infty$, and obtain

$$
M_{n, n-1}=\lambda^{2}
$$


The probability of transition $M_{n, n}$ becomes, after taking the limit $N \rightarrow$ $\infty$,

$$
\begin{aligned}
M_{n, n} & =2 \lambda(1-\lambda)+\frac{1}{N-1}(n-1)\left(3 \lambda^{2}-2 \lambda\right) \\
& \rightarrow 2 \lambda(1-\lambda)
\end{aligned}
$$

The probability of transition $M_{n, n+1}$ is first obtained:

$$
\begin{aligned}
M_{n-1, n} & =(1-\lambda)^{2}+\frac{n-1}{N-1}\left(-1+4 \lambda-3 \lambda^{2}\right) \\
\text { after replecement } n & \rightarrow n+1 \text { becomes } \\
M_{n, n+1} & =(1-\lambda)^{2}+\frac{n}{N-1}\left(-1+4 \lambda-3 \lambda^{2}\right)
\end{aligned}
$$

and note that at the limit $N \rightarrow \infty$ it becomes

$$
M_{n-1, n} \sim(1-\lambda)^{2}
$$

We introduce these results in the expression for $P_{n}(t+1)$,

$$
\begin{aligned}
P_{n}(t+1)= & \lambda^{2} P_{n-1}(t) \\
& +2 \lambda(1-\lambda) P_{n}(t) \\
& +(1-\lambda)^{2} P_{n+1}(t)
\end{aligned}
$$

This is the result.

\subsection{Avalanches in the Bak Sneppen model}

The avalanches of the Bak-Sneppen model are defined, for example, in Paczuski, Bak, and Maslov (1995). de Boer, Jackson, and Wettig (1995) defines a $\lambda$-avalanche as: "an evolution taking place between two successive times where the number $n$ of sites lower than $\lambda$ vanishes".

To make practical this definition one considers that an avalanche has started $t$ temporal steps ago. This can be considered time 0 .

One defines the probability $Q_{n}(t)$ of having $n$ sites with barriers $x_{i}<\lambda$, conditioned by the situation that at $t$ there was no site less than $\lambda$.

The probability $Q_{n}(t)$ verifies the same equation like $P_{n}(t)$ but with the constraint

$$
M_{0, n} \rightarrow 0
$$


This means that the transition (matrix element) from the state with $n$ sites $<\lambda$ to the state with 0 sites less than $\lambda$ is zero. This condition eliminates the possibility that from the state with a non-zero number of sites less than $\lambda$ the system cannot evolve to the state with 0 such sites, since if this were possible the avalanche would be terminated.

We examine in what conditions the avalanche terminates at time $t$.

Consider the probability $Q_{n}(t-1)$ of having $n$ sites less than $\lambda$ at time $t-1$.

The update from $t-1$ to $t$ replaces two sites: the lowest $x_{k}$ and the interacting $x_{l}$, chosen at random. The condition that the avalanche terminates at $t$ is that there is no more a site lower than $\lambda$, so the transition from $x_{k}<\lambda$ at $t-1$ to $x_{k}^{\prime}$ should move it to higher than $\lambda$ values. The probability is $(1-\lambda)$.

Now we recognize that there may be two initial states.

\subsubsection{Case A.}

The initial state, at $t-1$ consists of only one site less than $\lambda$ and this inevitably is the lowest, $x_{k}$. After update it will move to $>\lambda$ with probability $(1-\lambda)$.

However this is not all.

According to the algorithm we have to update another site, the one which is in interaction with $x_{k}$. This site, $x_{l}$ necessarly is NOT in the region $<\lambda$ as assumed. Then it is in the region $>\lambda$ but it makes a transition in the same region. This is with probability $(1-\lambda)$.

The probability of transition in this case $\mathrm{A}$ is

$$
(1-\lambda)^{2}
$$

The initial state was

$$
Q_{1}(t-1)
$$

\subsubsection{Case B}

The initial state at $t-1$ consists of two sites, which necessarly are

- the lowest, $x_{k}$ which after update goes to $>\lambda$ with probability $(1-\lambda)$;

- the secondary or interacting:

- the probability to chose (at random) the secondary one is uniform over the $N-1$ sites which - possibly - interact with $k$. 
- after update will move to $>\lambda$, with probability $(1-\lambda)$.

It results that in the case B we have the result

$$
\frac{1}{N-1}(1-\lambda)
$$

The initial state was described by the function

$$
Q_{2}(t-1)
$$

There is no other case. Since we canot assume the existence of more than two sites $<\lambda$ since we have chosen that only two sites are updated : the lowest one and the secondary, interacting.

Summing over the two cases

$$
\begin{aligned}
q(t)= & (1-\lambda)^{2} Q_{1}(t-1) \\
& +\frac{(1-\lambda)^{2}}{N-1} Q_{2}(t-1)
\end{aligned}
$$

The numerical simulation of this algorithm has led to the result

$$
q(t) \sim \frac{1}{t^{3 / 2}}
$$

and is also derived analytically by Paczuski. Maslov, and Bak (1996), de Boer, Jackson, and Wet (1995), de Boer, Derrida, Flyvbjerg, Jackson, and Wettig (1994).

\subsection{The limit of a large number of sites}

This means to take

$$
N \rightarrow \infty
$$

For this we re-examine the equations for $P_{n}(t)$.

\subsubsection{Calculation of the probabilities $P_{n}(t)$ for $N \rightarrow \infty$ and $t \rightarrow \infty$.} Connection with random walk with reflection

In the work of de Boer, Derrida, Flyvbjerg, Jackson, and Wettig (1994) it is calculated the probabilities $P_{n}$ for the large time limit. The reference model is a Random Walk with reflection at $n=0$. 
For the convergence of the geometric sum it is assumed

$$
\lambda<\frac{1}{2}
$$

Then the results are

$$
\begin{aligned}
& P_{0}=1-2 \lambda \\
& P_{1}=(1-2 \lambda)\left[\frac{1}{(1-\lambda)^{2}}+1\right] \\
& P_{n}=(1+2 \lambda) \frac{\lambda^{2 n-2}}{(1-\lambda)^{2 n}}
\end{aligned}
$$

They note that as

$$
\lambda \rightarrow \frac{1}{2}
$$

all probabilities vanish and this means that $n$ cannot remain finite. The probability that $n$ is finite and not very close to zero is vanishingly small. In physical terms this means that there will be no sites which have the fitness value under $\lambda=1 / 2$. This is the expression of the fact that the domain of fitness values under $\lambda=1 / 2$ is now effectively empty.

The same final conclusion is reached after calculating the probabilities $P_{n}(t)$ for $t \rightarrow \infty$ at $\lambda>1 / 2$.

The probability that an avalanche starts at 0 and ends at $t$ is

$$
q(t)
$$

This is calculated by first obtaining the probabilities (mentioned before) $Q_{n}(t)$ that, with an avalanche started at time 0 , there are at moment $t$ a number of $n$ sites that are still under $\lambda$.

$$
\begin{aligned}
& Q_{1}(1)=2 \lambda(1-\lambda) \\
& Q_{2}(1)=\lambda^{2} \\
& Q_{n}(1)=0 \text { for } n \geq 3
\end{aligned}
$$

The result is

$$
Q_{n}(t)=\frac{2 n(2 t+1) !}{(t+n+1) !(t-n+1) !} \lambda^{t+n-1}(1-\lambda)^{t-n+1}
$$

Now it is possible to calculate the probability that an avalanche has duration $t$ :

$$
q(t)=\frac{(2 t) !}{(t+1) ! t !} \lambda^{t-1}(1-\lambda)^{t+1}
$$


The average duration of an avalanche is

$$
\langle t\rangle=\sum_{t=1}^{\infty} t q(t)=\frac{1}{1-2 \lambda}
$$

and we see that it diverges for $\lambda \rightarrow 1 / 2$.

For large $t$ the probability of an avalanche of duration $t, q(t)$ has the asymptotic form

$$
q(t) \sim \frac{(1-\lambda)[4 \lambda(1-\lambda)]^{t}}{\lambda \sqrt{\pi}} \frac{1}{t^{3 / 2}}
$$

with the limit at $\lambda \rightarrow 1 / 2$ given by

$$
q(t) \sim \frac{1}{\tau^{3 / 2}}
$$

which is taken as the basis for the comparison with the statistics of the observations.

\section{References}

Arakawa, A. and Schubert, W. H.: Interaction of a cumulus cloud ensemble with the large-scale environment, Part I, Journal of the Atmospheric Sciences, 31, 674-701, 1974.

Bak, P.: How nature works: the science of self-organized criticality, vol. 212, Copernicus New York, 1996.

Bengtsson, L., Steinheimer, M., Bechtold, P., and Geleyn, J.: A stochastic parameterization for deep convection using cellular automata, Q. J. R. Meteorol. Soc., 139, 1533-1543, 2013.

Boettcher, S. and Paczuski, M.: Exact results for spatio-temporal correlations in a self-organized critical model of punctuated equilibrium, Phys. Rev. Lett., 76, 348-351, 1996.

Cruz, L.: Venezuelean rainstorms as seen by radar, J. Appl. Meteorol., 12, 119-126, 1973.

de Boer, J., Derrida, B., Flyvbjerg, H., Jackson, A. D., and Wettig, T.: Simple model of self-organized biological evolution, Phy. Rev. Lett., 73, 906-909, 1994. 
de Boer, J., Jackson, A. D., and Wettig, T.: Criticality in simple models of evolution, Phy. Rev. E, 51, 1059-1074, 1995.

Emanuel, K. A.: An airsea interaction model of intraseasonal oscillation in the tropics, J. Atmos. Sci., 44, 2324-2340, 1987.

Ito, K.: Punctuated-equilibrium model of biological evolution is also a selforganized-criticality model of earthquakes, Phys. Rev. E, 52, 3232-3233, 1995.

Jensen, H. J.: Self-organized criticality: emergent complex behavior in physical and biological systems, vol. 10, Cambridge university press, 1998.

Leary, C. and Houze Jr, R.: Structure and evolution of convection in a tropical cloud cluster, J. Atmos. Sci., 36, 437-457, 1979.

Lopez, R.: The lognormal distribution and cumulus cloud populations, Mon. Wea. Rev., 105, 865-872, 1977.

Mapes, B.: Gregarious tropical convection, J. Atmos. Sci., 50, 2026-2037, 1993.

Meinhardt, H.: Models of biological pattern formation, Academic Press, London, 1982.

Neelin, J., Held, I., and Cook, K.: Evaporation-wind feedback and lowfrequency variability in the tropical atmosphere, J. Atmos. Sci., 44, 23412348, 1987.

Paczuski, M., Bak, P., and Maslov, S.: Laws for stationary states in systems with extremal dynamics, Phys. Rev. Lett., 74, 4253-4256, 1995.

Paczuski, M., Maslov, S., and Bak, P.: Avalanche dynamics in evolution, growth and depinning models, Phys. Rev. E, 53, 414-443, 1996.

Peters, O. and Neelin, J. D.: Critical phenomena in atmospheric precipitation, arXiv.org cond-mat, 0606076, 1-5, 2006.

Peters, O., Hertlein, C., and Christensen, K.: A Complexity View of Rainfall, Phys. Rev. Lett., 88, 018 701, 2001.

Peters, O., Hertlein, C., and Christensen, K.: A Complexity View of Rainfall, Physical Review Letters, 88, 18 701, 2002.

Peters, O., Neelin, J. D., and Nesbitt, S.: Mesoscale convective systems and critical clusters, J. Atmos. Sci., 66, 2913-2924, 2009. 
Plant, R. and Craig, C.: A stochastic parameterization for deep convection based on equilibrium statistics, J. Atmos. Sci., 65, 87-105, 2008.

Sornette, D.: Critical phenomena in natural sciences, Springer series in synergetics, Springer, 2006.

Su, H., Bretherton, C., and Chen, S.: Self-aggregation and large-scale control of tropical deep convection: a modeling study, J. Atmos. Sci., 57, 17981816, 2000.

Yano, J.: Formulation structure of the mass-flux convection parameterization, Dyn. Atmos.Oceans, submitted. 